\title{
Growth of unstable interfaces in disordered media
}

\author{
A. M. Lacasta, ${ }^{1}$ L. Ramírez-Piscina, ${ }^{1}$ J. Casademunt, ${ }^{2}$ A. Hernández-Machado,${ }^{2}$ and M. A. Rodríguez ${ }^{3}$ \\ ${ }^{1}$ Departament de Física Aplicada, Universitat Politècnica de Catalunya, Avinguda Doctor Marañon 44, E-08028 Barcelona, Spain \\ ${ }^{2}$ Departament ECM, Facultat de Física, Universitat de Barcelona, Diagonal 647, E-08028 Barcelona, Spain \\ ${ }^{3}$ Departamento de Física Moderna, Universidad de Cantabria and Instituto de Física de Cantabria, \\ CSIC-UC, Avenida Los Castros, E 39005 Santander, Spain
}

(Received 1 July 1997)

\begin{abstract}
The effects of a disordered medium in the growth of unstable interfaces are studied by means of two local models with multiplicative and additive quenched disorder, respectively. For short times and large pushing the multiplicative quenched disorder is equivalent to a time-dependent noise. In this regime, the linear dispersion relation contains a destabilizing contribution introduced by the noise. For long times, the interface always gets pinned. We model the systematics of the pinned shapes by means of an effective nonlinear model. These results show good agreement with numerical simulations. For the additive noise we find numerically that a depinning transition occurs. [S1063-651X(98)04105-1]
\end{abstract}

PACS number(s): 68.10.Gw, 05.40.+j, 68.35.Ct

\section{INTRODUCTION}

The growth of interfaces in disordered media is an interesting nonequilibrium phenomenon. An example that has been studied extensively is the growth of a rough, macroscopically stable interface [1-4]. The aim of this paper is to study the growth of a morphologically unstable interface propagating through a disordered medium.

From the experimental point of view, the study of rough (stable) interfaces has been done, for example, in fluid flow in Hele-Shaw cells [5-7], paper wetting [8], propagation of burning fronts [9], and growth of bacterial colonies [10]. Our interest here would be in the morphologically unstable situation where fingers are developed at the macroscopic scale. Specifically, we focus on how the properties of the disorder at much smaller length scales affect the formation and dynamics of these fingered structures. Some controlled perturbations on the growth of unstable interfaces have been studied mostly experimentally. For a recent review of these studies on Hele-Shaw flow, see Ref. [11], and references therein. Similarly, a random perturbation introduced to the cell gap could be a simple model of unstable growth in the presence of a disordered medium. This possibility has already been explored experimentally [12]. In this case the disorder of the medium under consideration is frozen in time (quenched disorder).

From a theoretical point of view, the study of the growth of rough, macroscopically stable, interfaces has received much attention in recent years [13-19]. The theoretical approach to the case of disordered media has been mostly based on the quenched Edward-Wilkinson (QEW) [16] and quenched Kardar-Parisi-Zhang (QKPZ) equations [15]. These are local models with a diffusive term which accounts for surface tension effects and a constant force field which accounts for an external drift. In all cases the interface is rough and a depinning transition from pinned to moving regimes is found at a critical force $F_{c}$. In the moving regime, far above the critical force, the quenched noise behaves like a thermal noise recovering the classical EW and KPZ re- gimes $[13,14]$. On the other hand, below $F_{c}$ the whole interface becomes pinned. Recently the analysis of roughness close to the depinning transition has attracted the attention of researchers, both from experimental [5-10] and theoretical [15-19] points of view, since new universality classes of growth appear. The depinning transition is a phase transition characterized by a critical force and a diverging length of the pinning clusters. Just above the critical force the whole interface is moving but there are clusters with a characteristic length remaining pinned during a finite time.

Local models, such as the geometrical model $[20,21]$ and the boundary layer model [22], have also been proposed to study morphological instabilities like the ones observed in solidification patterns [23]. These local models are tractable simplifications of the complex nonlocal dynamics and provide a qualitative description of some aspects of the dynamic evolution. In the same spirit, and in order to study the effect of a quenched disorder in the growth of an unstable interface in the simplest possible situation, we propose two local models, with multiplicative and additive quenched disorder. They contain both surface tension and a constant external drift and should be considered as an attempt to elucidate the interplay between the deterministic instability, the quenched disorder, and the propagation of the interface.

The first model is defined by the following equation for the interface position $h(x, t)$ :

$\partial_{t} h(x, t)=-a \nabla^{2} h(x, t)-b \nabla^{4} h(x, t)+F+\eta(x, h) \nabla^{2} h(x, t)$.

The quenched disorder is introduced as spatial fluctuations of the external parameter $a$, and therefore has a multiplicative character. These fluctuations are defined on a square lattice of mesh size $\Delta x$ by a static noise that takes a different value in each cell. The noise values $\eta\left(x_{i}, y_{j}\right) \equiv \eta_{i j}$ are assumed to be Gaussian with zero mean and a correlation given by 


$$
\left\langle\eta_{i j} \eta_{k l}\right\rangle=2 \epsilon D_{i j k l},
$$

where $\epsilon$ is the noise intensity and $D_{i j k l}$ is a short-range correlation function. A quenched multiplicative noise has been introduced in a model of kinetic roughening [24] to consider an experimental situation in which the interfacial growth is dominated by pinning forces. Here, we propose Eq. (1.1) as the simplest model one can devise which is local, exhibits a deterministic instability, and modifies the linear dispersion relation with respect to the deterministic one.

The first term in the right hand side of Eq. (1.1) corresponds to the destabilizing effect of the external driving force which keeps the system out of equilibrium. The second term accounts for the surface tension stabilizing effect. The interplay between both effects generates the scale at which unstable fingers emerge. In the absence of noise, the constant pushing force $F$ fixes an average interface velocity which corresponds to that of the planar, unperturbed interface. Note that such velocity is taken as independent of the actual driving force of the instability (undercooling in solidification, injection rate in Hele-Shaw flows, etc.), which should appear in the coefficient $a$.

From this model we obtain two basic results. On the one hand we show how the quenched disorder behaves like a dynamic noise provided the interface is fast enough. It is known that a dynamic noise may change the dispersion relation in the case of multiplicative noise [25]. Here we can obtain analytically the change in the averaged linear dispersion relation in good agreement with numerical simulations. On the other hand we analyze the pinning phenomena in the presence of an instability. We find that the noise is surprisingly capable of stopping the otherwise exponential growth producing the pinning of the interface. This occurs in all our simulations in a time which diverges when the noise intensity goes to zero. We show that this surprising effect of the quenched noise is equivalent to the effect produced by a nonlinear term which conserves the symmetries of the original system.

As a second model, we introduce an additive quenched noise associated to fluctuations in the parameter $F$,

$$
\partial_{t} h(x, t)=-a \nabla^{2} h(x, t)-b \nabla^{4} h(x, t)+F+\eta(x, h),
$$

where $\eta(x, h)$ has the same properties as $\eta(x, h)$ of Eq. (1.1) but with noise intensity $\epsilon^{\prime}$. In Ref. [26] the model given by Eq. (1.3) with $a=0$ and a dynamic noise $\eta(x, t)$ has been discussed in connection with desorption in vapor deposition of solid films in the presence of gravity. Here, we consider the unstable situation $a \neq 0$ with quenched disorder. In this case we have found a behavior similar to that in the general case of stable growth [1-4], namely, that a depinning transition is always present. Close to the depinning transition there appear simultaneously regions with a pinned interface and regions with unstable growth [2]. When approaching the critical force from above the size of the pinning regions increases, but contrarily to what happens in the stable case now the pinning regions remain fixed and one finds quenched fingers coexisting with exponentially growing ones.

\section{LINEAR INSTABILITY AND MULTIPLICATIVE QUENCHED DISORDER}

The aim of this section is to show that, for large pushing, our model of multiplicative quenched disorder [Eqs. (1.1) and (1.2)] is equivalent to a model with a dynamic, timedependent, noise. For this reason, we obtain the dispersion relation associated to the multiplicative noise as the one of the dynamic noise, and we check this result by numerical integration of the model with multiplicative quenched disorder.

We consider the destabilization of an initially planar interface when pushed by the force $F$ through the disordered medium represented by the quenched noise $\eta(x, h)$. In this situation and at early times, far from a pinned situation, the whole interface moves at a velocity roughly equal to the value of $F$, encountering different values of the noise $\eta(x, h)$ at a rate given by the velocity $F$ and the correlation length of the noise $\lambda$. In fact one could say that the interface is affected by the values of the noise with an effective correlation time $\tau=\lambda / F$. Therefore, in this initial regime, the effects of the quenched noise are similar to what would be obtained if a dynamic noise $\xi(x, t)$ were acting on the system. This conclusion has also been obtained for models of rough interfaces [27] and seems to be general. We shall make explicit this analogy by using an equivalent model given by the discrete equation

$$
\partial_{t} h_{i}(t)=-a \nabla_{i j}^{2} h_{j}(t)-b \nabla_{i j}^{4} h_{j}(t)+\xi_{i}(t) \nabla_{i j}^{2} h_{j}(t),
$$

where the dynamic noise $\xi_{i}(t)$, in the limit of small $\tau$ $=\lambda / F$, is a white noise with correlation given by

$$
\left\langle\xi_{i}(t) \xi_{j}\left(t^{\prime}\right)\right\rangle=2 \bar{\epsilon} \bar{D}_{i j} \delta\left(t-t^{\prime}\right) .
$$

The explicit relation between $\epsilon$ and $\bar{\epsilon}$ depends on the particular properties of the noise [Eq. (1.2)]. We start by decoupling vertical and horizontal directions in Eq. (1.2) as $D_{i j k l}=\bar{D}_{i k} \bar{D}_{j l}$, in order to write the vertical direction as a temporal dependence.

We have considered two distinct particular cases. In the first one we take the correlation function of the noise as a smooth symmetric function $\bar{D}_{i k}=\bar{D}\left(r_{i k}\right)$ with $r_{i k}=\Delta x(k$ $-i)$ and $\int_{-\infty}^{\infty} d r \bar{D}(r)=1$. In the second case we have considered a completely uncorrelated discrete disorder. This situation, chosen here because it is the simplest way in which disorder can be implemented in a simulation, is defined by the discrete correlation function $D_{i j k l}=\left[1 /(\Delta x)^{2}\right] \delta_{i k} \delta_{j l}$, or equivalently

$$
\bar{D}_{i k}=\frac{1}{\Delta x} \delta_{i k}
$$

In this case the interface is affected by constant independent values of the noise in boxes of length $\Delta x$, that is, the correlation function as a function of the time difference has a triangular shape 


$$
\bar{D}\left(\left(t-t^{\prime}\right) F\right)=\left\{\begin{array}{cc}
\frac{1}{\Delta x}\left(1-\left|t-t^{\prime}\right| \frac{F}{\Delta x}\right) & \text { if } \quad\left|t-t^{\prime}\right| F<\Delta x \\
0 & \text { otherwise. }
\end{array}\right.
$$

In both cases, in the limit of small $\lambda / F$ (small $\Delta x / F$ for uncorrelated cells) this correlation function can be written as a function of the time difference as

$$
\bar{D}\left(\left(t-t^{\prime}\right) F\right)=F^{-1} \delta\left(t-t^{\prime}\right) .
$$

In view of this equation, and comparing Eqs. (1.2) and (2.2), we have

$$
\epsilon=F \bar{\epsilon} .
$$

The next step is to write the equation obeyed by the mean (over realizations of the noise) of the interface position $\left\langle h_{i}(t)\right\rangle$. To do that one has to evaluate the mean value of the noise term in Eq. (2.1). We employ Novikov's theorem [28], which gives the mean of the product of the noise and any functional $\psi$ of the noise:

$$
\left\langle\xi_{i}(t) \psi(t)\right\rangle=\int_{0}^{t} d t^{\prime} \sum_{j}\left\langle\xi_{i}(t) \xi_{j}\left(t^{\prime}\right)\right\rangle\left\langle\frac{\delta \psi(t)}{\delta \xi_{j}\left(t^{\prime}\right)}\right\rangle .
$$

We obtain

$$
\left\langle\xi_{i}(t) \nabla_{i j}^{2} h_{j}(t)\right\rangle=\bar{\epsilon} \bar{D}_{i j}\left\langle\left(\nabla_{i j}^{2} \nabla_{j k}^{2} h_{k}(t)\right\rangle .\right.
$$

In the smooth correlation function case, we can expand $\bar{D}$ by Taylor as $\bar{D}_{i j}=\bar{D}(0)+\frac{1}{2} \bar{D}^{\prime \prime}(0) r_{i j}^{2}+o\left(r_{i j}^{4}\right)$. Then the mean of Eq. (2.8) can be written as a continuous equation (the discrete Laplacian operator $\nabla_{i j}$ is of order $\Delta x^{-2}$ and nonzero only for $r_{i j}$ up to the order of $\Delta x$ ) and reduces to

$$
\begin{aligned}
\left\langle\xi(x, t) \nabla^{2} h(x, t)\right\rangle= & \bar{\epsilon} \bar{D}^{\prime \prime}(0)\left\langle\nabla^{2} h(x, t)\right\rangle \\
& +\bar{\epsilon} \bar{D}(0)\left\langle\nabla^{4} h(x, t)\right\rangle
\end{aligned}
$$

and the equation for the first moment of $h(x, t)$ is

$$
\begin{aligned}
\partial_{t}\langle h(x, t)\rangle= & -[a-\bar{\epsilon} \bar{D}(0)]\left\langle\nabla^{2} h(x, t)\right\rangle \\
& -\left[b-\bar{\epsilon} \bar{D}^{\prime \prime}(0)\right]\left\langle\nabla^{4} h(x, t)\right\rangle .
\end{aligned}
$$

Now, the linear dispersion relation can be easily derived by defining the growth rate for a mode $k, \omega_{k}$, as $\langle\hat{h}(k, t)\rangle$ $=\langle\hat{h}(k, 0)\rangle e^{\omega_{k} t}$. We obtain in terms of the intensity of the original quenched noise

$$
\omega_{k}=\left(a-\frac{\epsilon}{F} \bar{D}(0)\right) k^{2}-\left(b-\frac{\epsilon}{F} \bar{D}^{\prime \prime}(0)\right) k^{4} .
$$

The first term is stabilizing and the second one is destabilizing. Provided that $\bar{D}^{\prime \prime}(0)<0$ the presence of the noise makes the interface more unstable.

A similar conclusion will be obtained in the second case addressed here, namely, the case of a completely uncorre-

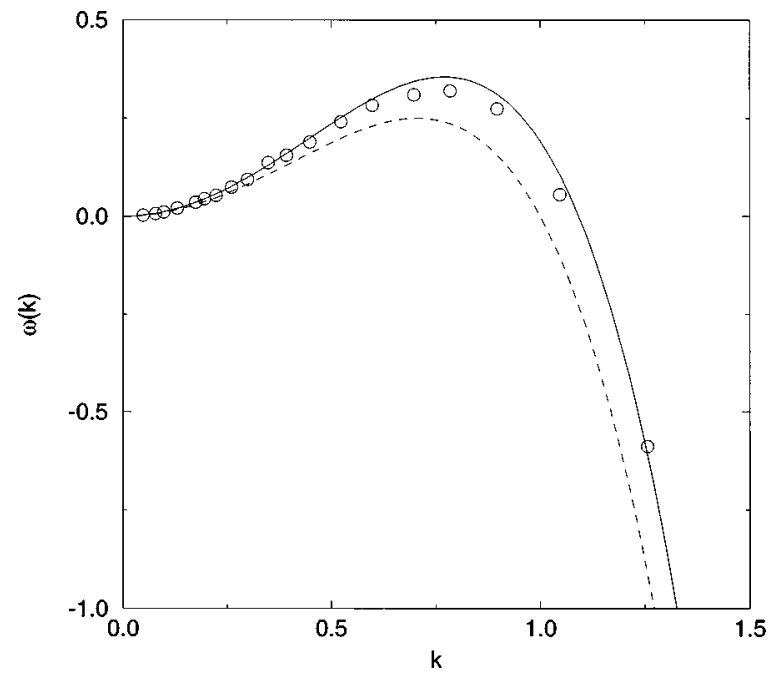

FIG. 1. Dispersion relation from the simulation results of Eqs. (1.1), (1.2), and (2.3) ( $\bigcirc)$ with $F=1000$ and $\epsilon=1.5$, compared to the analytical expression of Eq. (2.13) (solid line) with $\bar{\epsilon}=1.5$ $\times 10^{-3}$. The dashed line corresponds to the results for the deterministic case $\bar{\epsilon}=0$.

lated disorder. By using Eqs. (2.8) and (2.3), the equation for the first moment in this situation is

$$
\partial_{t}\left\langle h_{i}(t)\right\rangle=-\left(a+2 \frac{\bar{\epsilon}}{(\Delta x)^{3}}\right)\left\langle\nabla^{2} h_{i}(t)\right\rangle-b\left\langle\nabla^{4} h_{i}(t)\right\rangle .
$$

In this expression we have taken for the Laplacian operator $\nabla_{i j}^{2}=\Delta x^{-2}\left(\delta_{i j-1}-2 \delta_{i j}+\delta_{i j+1}\right)$, where $\Delta x$ is the mesh size. Finally, the dispersion relation reads

$$
\omega_{k}=\left(a+\frac{2 \epsilon}{F(\Delta x)^{3}}\right) k^{2}-b k^{4} .
$$

Therefore we see that also in this particular discretization scheme with uncorrelated fluctuations the noise acts to make the interface more unstable.

To corroborate the equivalence between quenched and dynamic noise, we have performed the numerical integration of Eqs. (1.1), (1.2), and (2.3) and compared the resulting dispersion relation with the prediction from Eqs. (2.13) and (2.6). We have used a square lattice with independent noise values in each cell, and the parameter values $a=b=1$ and $\Delta x=0.25$. In Fig. 1 we have plotted the dispersion relation from the simulation results compared to the prediction of Eqs. (2.13) and (2.6). The agreement is quite good. We also show the deterministic case with a dashed line.

\section{LARGE AMPLITUDES AND INTERFACE PINNING FOR MULTIPLICATIVE QUENCHED DISORDER}

In this section we study the long time behavior of the interface for the multiplicative quenched noise. The basic point is that when unstable modes have grown to large amplitudes, some regions of the interface will inevitably attain small local velocities. The quenched disorder case becomes radically different from both the deterministic and the dy- 


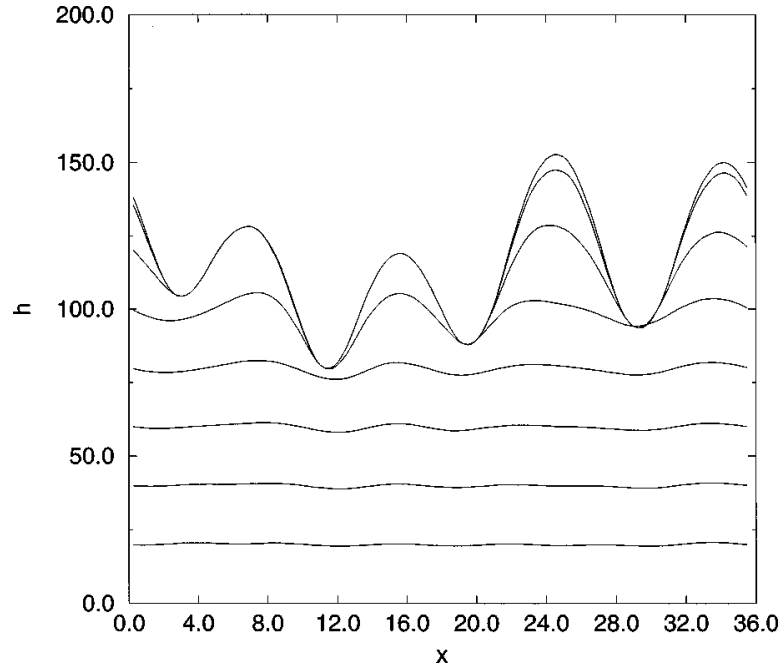

FIG. 2. Time sequence of the interfacial evolution $(t$ $=2,4, \ldots, 20)$ for the same model of Fig. 1 with $\epsilon=1.25$ and $F$ $=10$.

namic noise cases. As a consequence, in those regions the disorder cannot be modeled by a dynamic noise. The fundamental difference lies in the fact that with a quenched disorder, and at least for the range of noise intensities studied here, the interface always gets pinned for large enough times. The pinning of the interface does not occur simultaneously everywhere but rather starts in the regions where the interface velocity is close to vanishing. In such regions it will be likely to find local values of the noise which will pin the interface. Then, the pinned domains will propagate to cover the whole interface. The particular location where the interface gets pinned depends on the noise realization but the final shape of the interface has some interesting systematics.

\section{A. Numerical results}

In Fig. 2 we show a generic time sequence of the interface evolution for a large noise intensity. The horizontal size of the system is $L=142 \Delta x$, with $\Delta x=0.25$. When the interface is almost flat, it advances with a constant velocity. When the most unstable modes grow to appreciable amplitudes, the local minima of $h$ eventually attain small velocities and get pinned. After a short time the pinned region reaches the fastest tips, which also stop completely. For large noise intensities the pinning process seems to start systematically at the local minima. The final configuration of the interface (location of extremes) is rather sensitive to the details of the noise realization. The curvature of the maxima and the minima, however, seems to be always very similar. We will come back to this point later on. In Fig. 3 we show a similar run but now for a smaller noise intensity. Here we see that the amplitudes at which pinning occurs are much larger. Also, one sees that the pinning of the interface does not start at the minima of the interface.

In Fig. 4 we have plotted a superposition of different runs with the same values for both parameters $\epsilon$ and $F$ but with different initial conditions and noise realization. The two runs, one with random initial condition and the other with sinusoidal initial profile, gave apparently different outcomes for the final pinned shape. We have relocated the interfaces

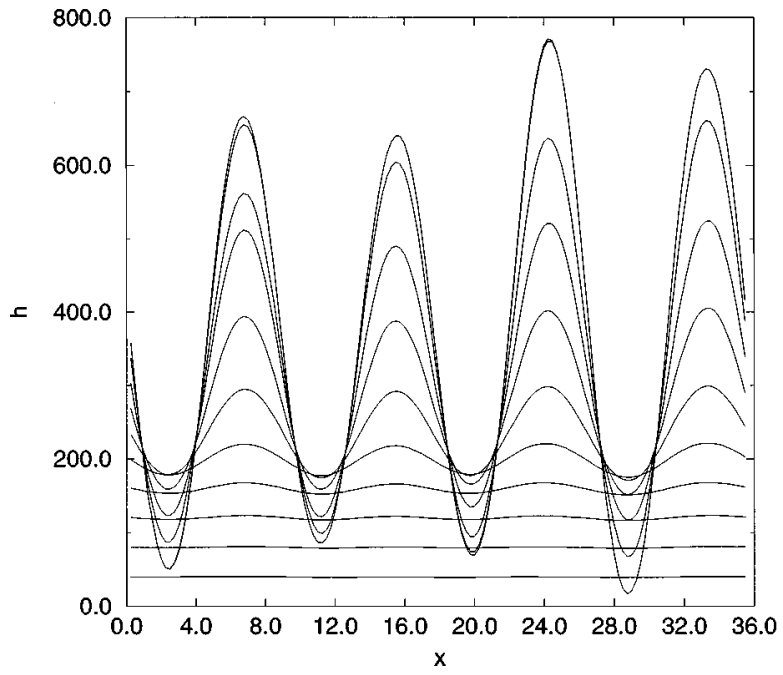

FIG. 3. Same as Fig. 2 with $\epsilon=0.25$ and $t=4,8, \ldots, 60$.

so that two local maxima and two local minima of the two curves, respectively, coincide. We see that the two curves in the maxima region match quite remarkably. This is not a coincidence but a quite systematic observation. A similar fact occurs for the minima, although the curvatures do not seem to match as well as for the maxima.

In Fig. 5(a) we plot the dependence of the curvature at the maxima versus the parameter $F$. The dependence is linear within a very good approximation. In Fig. 5(b) we also plot the slope of the fitted lines of Fig. 5(a) versus $1 / \epsilon$. In this case the dependence seems to be less clear.

\section{B. Effective model}

Now, we propose an effective deterministic equation to model the shape of the pinned interfaces resulting from Eqs. (1.1), (1.2), and (2.3). From our numerical results and symmetry arguments, we conclude that, in the range of moderately large $\epsilon$ and for the values of $F$ studied, the effect of the

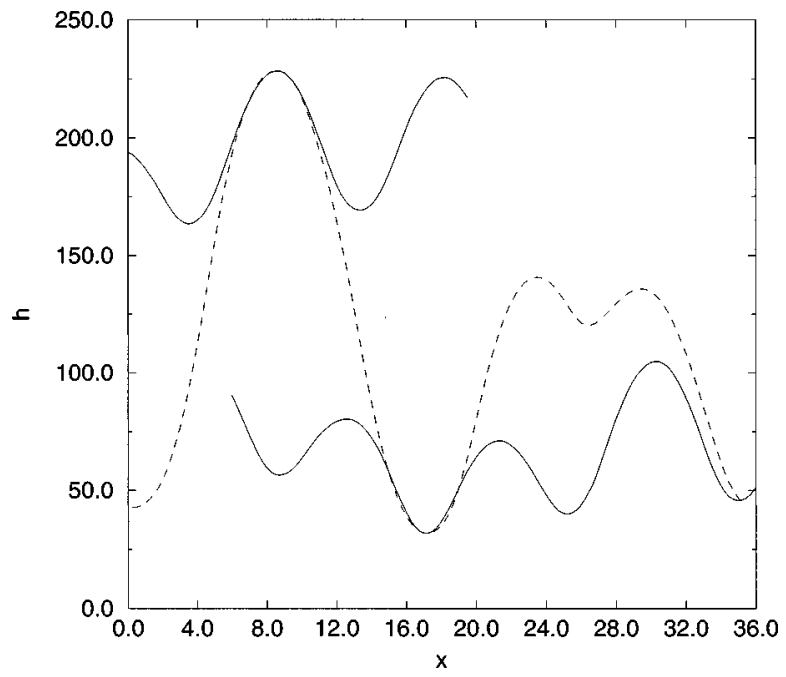

FIG. 4. Comparison of the maxima and minima of the final interfacial profile obtained from the simulation of Eqs. (1.1), (1.2), and (2.3) for two different initial conditions: random (solid line) and sinusoidal with two maxima (dashed line). 

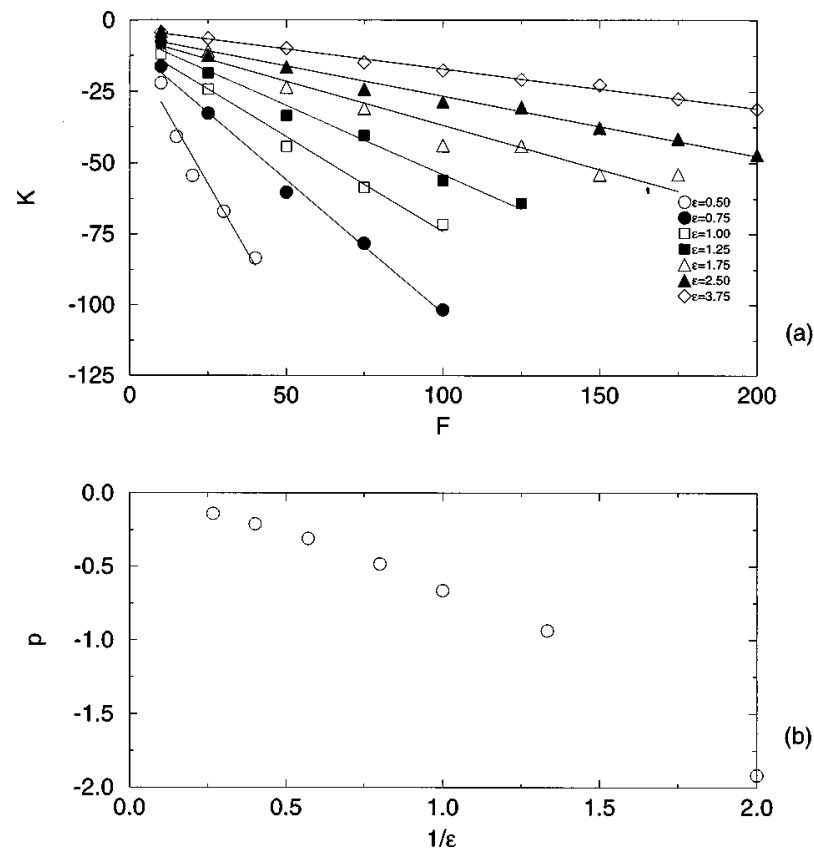

FIG. 5. (a) Curvature at the maxima of the final interfacial profile versus the parameter $F$, obtained from the simulation of Eqs. (1.1), (1.2), and (2.3) for different values of the noise intensity $\epsilon$.

(b) Slope $p$ of the fitted lines of (a) versus $1 / \epsilon$.

noise term can be described by an expansion in odd powers of the second derivatives of $h$. Keeping up to the lowest nonlinear term we get

$$
\begin{aligned}
\partial_{t} h(x, t)= & -\nabla^{4} h(x, t)-(1-\alpha) \nabla^{2} h(x, t) \\
& +\beta\left[\nabla^{2} h(x, t)\right]^{3},
\end{aligned}
$$

where the parameters $\alpha$ and $\beta$ are to be determined from the simulations.

In Fig. 6(a), by fitting the parameter $\beta$, with $\alpha=0$, we find that the effective model is qualitatively correct, compared with the results of the numerical integration of Eqs. (1.1), (1.2), and (2.3). However, to obtain a better quantitative result for the curvature [Fig. 6(b)] it is convenient to include the parameter $\alpha$. In this case, the stationary solution for the second derivative of Eq. (3.1) in an infinite system is

$$
\nabla^{2} h=\sqrt{\frac{1-\alpha}{\beta}} \tanh \sqrt{\frac{1-\alpha}{2}} x .
$$

Then, for a periodic system, the stationary solution for the second derivative consists of regions with values $\pm \sqrt{(1-\alpha) / \beta}$ joined by hyperbolic tangents with a width that depends on the parameter $\alpha$. We note that only for a negative and large enough value of $\alpha$ can we have a parabolic structure for the interface near the minimum, with a curvature given by the limiting value of Eq. (3.2). In Figs. 6(a) and 6(b) we present the final interfacial profile and its second derivative obtained from the simulation of Eqs. (1.1), (1.2), and (2.3). We compare these results to the ones obtained from the effective model for $\alpha=0$ and $\alpha \neq 0$.

For $\alpha=0$ it is necessary to fit the parameter $\beta$ to obtain the correct value for the maximum, whereas for $\alpha \neq 0$ the result is calculated from Eq. (3.2). In this last case, we obtain

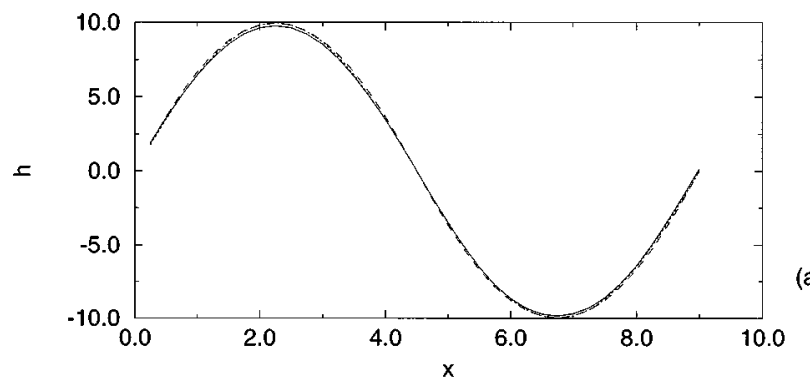

(a)

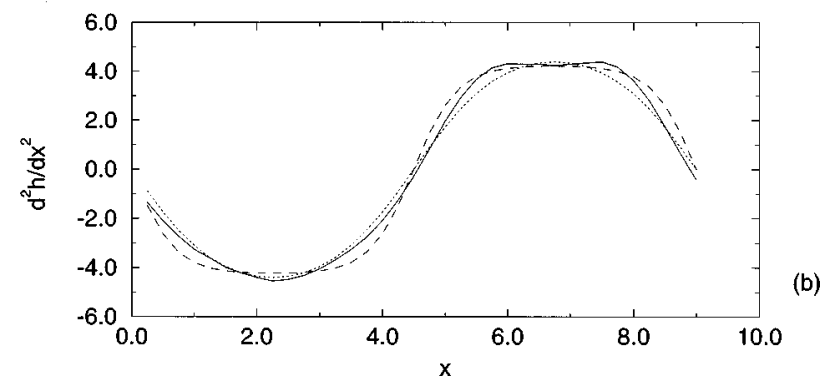

FIG. 6. (a) Final interfacial profiles from the simulation of Eqs. (1.1), (1.2), and (2.3) (solid line) compared to the results of the effective model [Eq. (3.1)] for $\alpha=0, \beta=0.03$ (dotted line) and for $\alpha=-3, \beta=0.22$ (dashed line). (b) Second derivative of the profiles for the cases of (a).

an approximately constant value for the second derivative around the minimum in accordance with the simulation results with the stochastic term.

\section{THE CASE OF ADDITIVE QUENCHED NOISE: A DEPINNING TRANSITION}

In this section we consider the case of quenched noise in the force field [Eq. (1.3)]. In the classical QEW and QKPZ models the force field provides both the pushing and pinning mechanisms for the growth of the interface. The surface tension effect coming from the other terms of the model only acts to smooth the roughness generated by the force field. In our case a new mechanism, the unstable growth of some selected modes, is present. The interplay between pinning and unstable growth is not well known and questions such as the existence of a depinning transition and the behavior near the critical point are very interesting.

In this approach we have treated these points numerically. We have simulated Eq. (1.3) keeping fixed the dispersion relation by varying only the parameters of the force field, the mean value $F$ and the intensity of the noise $\epsilon^{\prime}$. A transition from pinned to depinned regimes is always found. In Fig. 7 pinned and moving regimes are shown in the space parameter $F$ versus $\epsilon^{\prime}$. The depinning transition occurs for $F$ varying linearly with $\epsilon^{\prime}$ as $F=0.88 \epsilon^{\prime}-0.73$.

Near the depinning point there are regions of pinned and moving interfaces as shown in Fig. 8. Here it can be seen that the quenched disorder is capable of suppressing the growth of some fingers. Note that this mechanism of suppression is exclusively originated by the force field $F$ in contrast to other phenomena of suppression which require nonlinear effects giving rise to competitive processes of growth. We have observed that the length of the region in which fingers are suppressed is larger as $F$ approaches its 


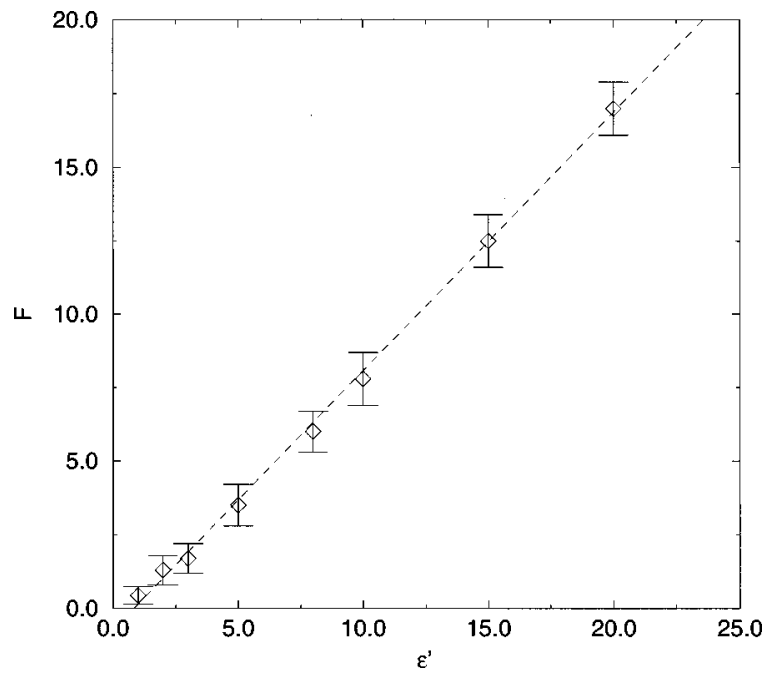

FIG. 7. Location of the depinning transition in the parameters space $F-\epsilon^{\prime}$ for the additive noise model [Eq. (1.3)]. The dashed line corresponds to the equation $F=0.88 \epsilon^{\prime}-0.73$.

critical value. These regions are the equivalent to the clusters of pinning observed in the QEW and QKPZ models but with the important difference that in our case the suppression regions are static while in the case of stable growth the pinning clusters change with time.

\section{CONCLUSIONS}

In this paper we have studied the effects of a disordered medium in the growth of unstable interfaces pushed by an external driving force $F$. We have used two local models with multiplicative and additive quenched noise, respectively. We have found that, for short time and large pushing, the multiplicative quenched noise is equivalent to a timedependent multiplicative noise. We have worked out explicitly the relation between the static and dynamic noise intensities. We have calculated the linear dispersion relation for two different types of spatial correlation of the disorder. We have found a destabilizing contribution of the quenched disorder. Furthermore, we have checked this result by numerical simulation in the case of a completely uncorrelated dis-

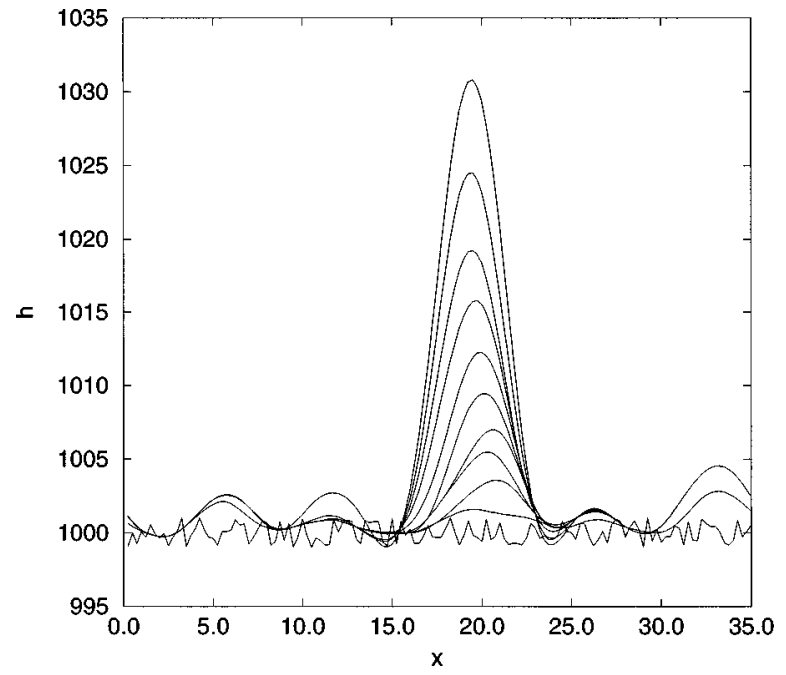

FIG. 8. Time sequence of the interfacial evolution for the additive noise case with $\epsilon^{\prime}=3$ and $F=1.7$.

crete static disorder. We have also studied the long time behavior of interfaces for a multiplicative quenched disorder. A remarkable result is that the interfaces always get pinned for large enough time in the absence of nonlinear effects other than those introduced by the disorder. The final profile near the maxima and minima is independent of the initial condition and the realization of the noise, and the curvature at these points has a linear dependence versus the parameter $F$. We have proposed an effective nonlinear deterministic equation to model the shape of the pinned interfaces. Finally, we have considered the case of additive noise. We have observed a depinning transition with a linear dependence between $F$ and the noise intensity $\epsilon^{\prime}$.

\section{ACKNOWLEDGMENTS}

We acknowledge financial support from Dirección General de Enseñanza Superior (Project Nos. PB96-0241-C0202, PB96-0378-C02-01, PB96-00378-C02-02, and PB961001-C02-02), and the Comissió Interdepartamental de Recerca i Innovació Tecnològica (Project No. 1995SGR00459). We also acknowledge computing support from the Center de Computació i Comunicacions de Catalunya.
[1] Dynamics of Fractal Surfaces, edited by F. Family and T. Vicsek (World Scientific, Singapore, 1991).

[2] A.-L. Barabási and H. E. Stanley, Fractal Concepts in Surface Growth (Cambridge University Press, Cambridge, England, 1995).

[3] J. Krug and H. Spohn, Solids Far From Equilibrium: Growth, Morphology and Defects, edited by C. Godreche (Cambridge University Press, Cambridge, England, 1991).

[4] T. Halpin-Healy and Y. C. Zhang, Phys. Rep. 254, 215 (1995).

[5] M. A. Rubio, C. A. Edwards, A. Dougherty, and J. P. Gollub, Phys. Rev. Lett. 63, 1685 (1989).

[6] V. K. Horvath, F. Family, and T. Vicsek, J. Phys. A 24, L25 (1991).

[7] S. He, G. L. M. K. S. Kahanda, and P.-z Wong, Phys. Rev. Lett. 69, 3731 (1992).
[8] L. A. N. Amaral, A.-L. Barabási, S. V. Buldyrev, S. Havlin, and H. E. Stanley, Phys. Rev. Lett. 72, 641 (1994).

[9] J. Zhang, Y.-C. Zhang, P. Alstrom, and M. T. Levinsen, Physica A 189, 383 (1992).

[10] E. Ben-Jacob, O. Shochet, A. Tenenbaum, I. Cohen, A. Czirok, and T. Vicsek, Fractals 2, 15 (1994).

[11] K. V. McCloud and J. V. Maher, Phys. Rep. 260, 139 (1995).

[12] J. Ignés-Mullol, J. Ortín, and J. V. Maher (private communication).

[13] S. F. Edwards and D. R. Wilkinson, Proc. R. Soc. London, Ser. A 381, 17 (1982).

[14] M. Kardar, G. Parisi, and C. Zhang, Phys. Rev. Lett. 56, 889 (1986).

[15] L. A. N. Amaral, A.-L. Barabási, and H. E. Stanley, Phys. Rev. Lett. 73, 62 (1994). 
[16] D. A. Kessler, H. Levine, and Y. Tu, Phys. Rev. A 43, 4551 (1991).

[17] N. Martys, M. Cieplak, and M. O. Robbins, Phys. Rev. Lett. 66, 1058 (1991).

[18] G. Parisi, Europhys. Lett. 17, 673 (1992).

[19] T. Nattermann, S. Stepanow, L.-H. Tang, and H. Leschhorn, J. Phys. II 2, 1483 (1992).

[20] R. C. Brower, D. A. Kessler, J. Koplik, and H. Levine, Phys. Rev. Lett. 51, 1111 (1983).

[21] D. A. Kessler, J. Koplik, and H. Levine, Phys. Rev. A 31, 1712 (1985).

[22] J. S. Langer, Phys. Rev. A 33, 435 (1986).
[23] P. Pelce, in Dynamics of Curved Fronts, Perspectives in Physics (Academic Press, New York, 1988).

[24] T. Vicsek, E. Somfai, and M. Vicsek, J. Phys. A 25, L763 (1992).

[25] L. Ramírez-Piscina, A. Hernández-Machado, and J. M. Sancho, Phys. Rev. B 48, 119 (1993).

[26] D. E. Wolf and J. Villain, Europhys. Lett. 13, 389 (1990).

[27] J. M. López, M. A. Rodríguez, A. Hernández-Machado, and A. Díaz-Guilera, Europhys. Lett. 29, 197 (1995).

[28] E. A. Novikov, Zh. Éksp. Teor. Fiz. 47, 1919 (1964) [Sov. Phys. JETP 20, 1290 (1965)]. 